\title{
Risque environnemental pour la maladie de Lyme dans l'est et le centre du Canada : un sommaire d'informations récentes en matière de surveillance
}

\author{
Ogden N. H. ${ }^{1^{*}}$, Koffi J. K. ${ }^{1}$, Pelcat Y. $^{2}$ \\ et Lindsay L. R. ${ }^{2}$ \\ ${ }^{1}$ Centre des maladies infectieuses d'origine alimentaire, environnementale et zoonotique, Agence de la santé publique \\ du Canada, Saint-Hyacinthe, Québec \\ ${ }^{2}$ Laboratoire national de microbiologie, Agence de la santé publique du Canada, Winnipeg, Manitoba \\ *Auteur-ressource : nicholas.ogden@phac-aspc.gc.ca
}

\section{Résumé}

Contexte : La maladie de Lyme est la maladie à transmission vectorielle la plus communément signalée en zone tempérée. Au Canada, elle émerge du fait de la propagation de la tique vectrice Ixodes scapularis dans le territoire et d'un endroit à l'autre pour former de nouvelles aires de risque environnemental que l'on appelle " aires endémiques de la maladie de Lyme ». II importe de localiser ces aires : cela permettra de recenser les populations à risque, de cibler les mesures à prendre et d'orienter le diagnostic clinique des patients atteints de la maladie.

Objectif : Fournir un portrait à jour des aires actuelles et émergentes de risque pour la maladie de Lyme dans l'est et le centre du Canada dans un sommaire de données récentes sur les aires endémiques de la maladie et sur la surveillance des tiques $I$. scapularis.

Méthodes : On a collecté et cartographié des données sur les endroits qui attestent la présence de $I$. scapularis selon des études de surveillance sur le terrain (effectuées par un éventail d'organismes fédéraux et provinciaux), afin d'obtenir un portrait plus complet de la présence de $I$. scapularis au Canada. De plus, ont été cartographiées à des fins de comparaison les aires géographiques de tiques soumises dans le cadre de la surveillance passive.

Résultats : On a vu s'accroître le nombre d'aires endémiques de la maladie de Lyme confirmées dans le sud du Manitoba, le sud et l'est de l'Ontario, le sud du Québec, le sud du Nouveau-Brunswick et dans certaines localités de la Nouvelle-Écosse; ces aires sont en effet passées de 10, en 2009, à 22, en 2012. D'après les données recueillies par la surveillance sur le terrain, les populations de tiques $I$. scapularis et le risque pour la maladie de Lyme sont plus géographiquement étendus que les aires endémiques connues; et la répartition de l'émergence de ces populations fluctue d'une province à l'autre. On a observé un décuplement du nombre de $I$. scapularis signalées par la surveillance passive : de 2059 soumissions pour la période 1990-2003 à 25 738, pour 20042012.

Conclusions : Le nombre croissant d'aires endémiques de la maladie de Lyme, la répartition sensiblement plus vaste des populations de tiques repérées par surveillance sur le terrain et la montée de flèche des tiques détectées par la surveillance passive : voilà trois réalités qui donnent à penser que s'étend dans l'est et le centre du Canada la portée géographique du risque environnemental pour la maladie de Lyme - mais ce ne sont que les parties sud de cinq provinces qui sont les plus touchées.

\section{Introduction}

La maladie de Lyme, induite par Borrelia burgdorferi, est la maladie à transmission vectorielle la plus communément signalée dans la zone tempérée, touchant ainsi notamment l'Amérique du Nord (1,2). Cette bactérie est transmise par les tiques, qui se nourrissent sur des hôtes fauniques réservoir, notamment les rongeurs et les oiseaux. L'espèce Ixodes scapularis, tique à pattes noires, en est la principale vectrice dans l'est 
et le centre de l'Amérique du Nord, alors que l'espèce Ixodes pacificus, tique occidentale à pattes noires, en est la grande vectrice à l'ouest des montagnes Rocheuses. Ces deux espèces de tiques choisissent indifféremment leur hôte et se nourrissent aussi sur les humains, d'où une transmission possible de l'agent pathogène de la faune à l'humain. La présente étude porte sur des données de surveillance des tiques Ixodes scapularis détectées dans l'est et le centre du Canada. Quant à l'espèce Ixodes pacificus, elle fait présentement l'objet d'études visant à obtenir des données sur sa présence dans l'Ouest canadien - données que l'on fera connaître dans des articles à venir.

Le risque d'infection à $B$. burgdorferi et à d'autres pathogènes transmis par $I$. scapularis (Anaplasma phagocytophilum, Babesia microti, Borrelia miyamotoi, une nouvelle bactérie apparentée à Ehrlichia muris, et le virus Powassan) $(3,4,5)$ s'accroît dans l'est et le centre du Canada, à mesure que se répandent des populations de $I$. scapularis vers le nord - à une vitesse estimative de 33 à $55 \mathrm{~km}$ par année (6).

Il est important de repérer les aires où les tiques sont en voie d'établissement au Canada, car la présence géographique de ces insectes permet de délimiter les aires à risque pour la maladie de Lyme à l'heure actuelle et à l'avenir (7). L'incidence annuelle des cas signalés de maladie de Lyme chez les humains s'est accrue de façon marquée au Canada ces dernières années (Figure 1). En effet, alors que 40 cas ont été signalés en 2004 au pays, ce nombre est passé à 315, en 2012. De telles données pourraient bien refléter une hausse du nombre de cas signalés, mais une sous-déclaration pourrait également se produire (8).

Dans le présent article, nous décrivons la localisation plausible des aires à risque pour la maladie de Lyme d'après trois sources : les données sur les aires endémiques de la maladie confirmées et présumées; les résultats d'une méthode simplifiée de surveillance sur le terrain visant à recenser les aires à risque émergentes; et la surveillance passive des tiques. Cette dernière technique comprend la soumission des insectes aperçus sur des patients de cliniques médicales et vétérinaires participantes. La détection des aires endémiques de la maladie de Lyme dans l'environnement est la référence comme technique de surveillance du risque; mais comme cette méthode requiert de multiples visites d'emplacements sur deux ans, elle n'est pas très opportune ou pratique. L'échantillonnage à filet traînant seul est plus pratique et rapide, mais offre une sensibilité et une spécificité moindres (9). Quant à la surveillance passive des tiques, il s'agit d'une méthode sensible pour la détection des tiques $I$. scapularis, mais elle se révèle relativement non spécifique eu égard à la localisation géographique des populations de tiques; cela, en raison de tiques moins nombreuses disséminées par les oiseaux migrateurs sur de longues distances à partir de populations de tiques. Les tiques ainsi disséminées se nomment « tiques adventices » (10).

\section{Figure 1 : Nombres de cas de la maladie de Lyme signalés au Canada de 1994 à 1012*}

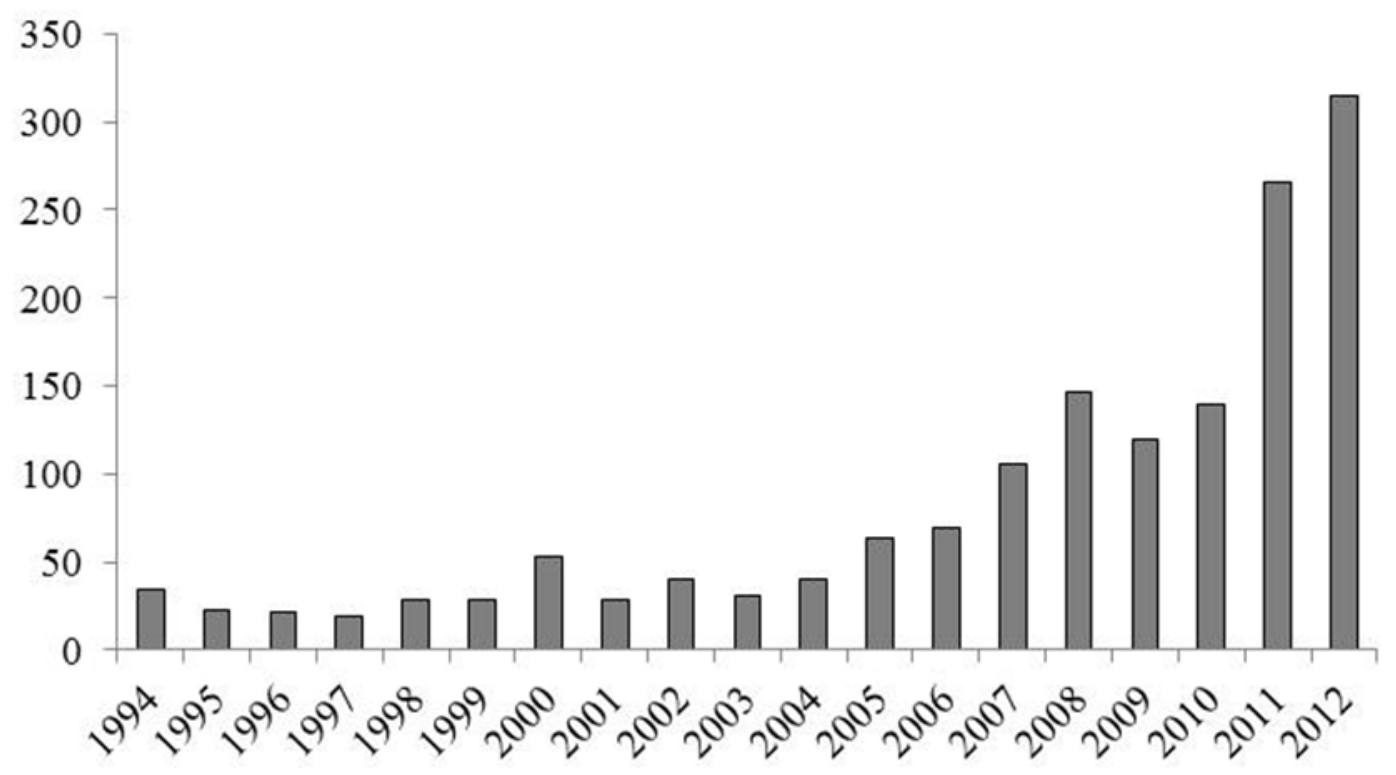


* Les nombres de cas antérieurs à 2009, année où la maladie de Lyme est devenue à déclaration obligatoire au

Canada, sont des estimations fondées sur une information issue des organismes provinciaux de santé publique (10).

Disposer d'information sur les endroits à risque constitue un atout à la fois pour les praticiens de santé publique qui tiennent à mieux cibler leurs activités de prévention et de lutte, pour les professionnels des soins de santé qui ont à cœur de meilleurs diagnostics de la maladie de Lyme et pour le public, qui pourra ainsi faire des choix plus éclairés en la matière. Pour ce qui est de la situation dans l'Ouest canadien, on attend présentement les résultats d'études sur le terrain en cours.

Quant à la présente étude, elle a pour objectif de cartographier nos connaissances actuelles des aires à risque pour la maladie de Lyme dans l'est et le centre du Canada selon trois grands axes :

- Collecte des connaissances sur les aires endémiques de la maladie de Lyme présentement connues;

- Délimitation des aires endémiques de la maladie de Lyme émergentes plausibles par la surveillance des tiques sur le terrain;

- Détection du risque que posent les tiques adventices par l'analyse des données de surveillance passive.

\section{Méthodes}

Trois types de données de surveillance des tiques ont été recueillies : données sur les aires endémiques de la maladie de Lyme - information qui fait appel à de vastes essais; données de surveillance sur le terrain menée entre 2008-2013; et données de surveillance passive entre 2004-2012.

\section{Aires endémiques de la maladie de Lyme}

Les aires endémiques de la maladie de Lyme sont des endroits où a été confirmée la transmission de $B$. burgdorferi par des populations résidentes de tiques vectrices (11). À cette fin, on a fait appel à deux méthodes : 1.) l'échantillonnage par filet de traînée (il s'agit de faire traîner sur le sol forestier de l'habitat un filet en flanelle d'un mètre carré pour au moins 3 heures-personnes/emplacement) pour recueillir des tiques en quête de sang en guise de nourriture; 2.) la capture de rongeurs fauniques qui sont des hôtes réservoir importants pour la bactérie. Afin de confirmer une aire endémique, il faut pouvoir y détecter les trois stades développementaux de la tique (la larve, la nymphe et l'adulte), ainsi que $B$. burgdorferi, pendant plus d'un an. Pour ce qui est des aires endémiques présumées, il s'agit d'endroits où ont été repérées des tiques à plus d'un stade, où a été dépistée $B$. burgdorferi sur des échantillons de tiques ou de rongeurs, mais où une seconde année d'échantillonnage sur le terrain n'a pas encore eu lieu. Toutes ces aires endémiques connues ou présumées, qui reposent sur des données fournies par des organismes de santé publique provinciaux, ont été cartographiées avec le logiciel ArcGIS Version 10.2 (ESRI).

\section{Surveillance sur le terrain}

Des données ont été recueillies attestant la présence de $I$. scapularis lors d'études sur le terrain menées par un personnel chevronné dans le cadre de collaborations entre l'Agence de la santé publique du Canada et des organismes gouvernementaux de cinq provinces : le Nouveau-Brunswick (2008), le Québec (2010-2012), le Manitoba (2010-2012), la Nouvelle-Écosse (2102) et l'Ontario (2012-2013). À ce matériel s'ajoutent des données de surveillance menée de 2010 à 2012 au moyen de l'échantillonnage par filet traînant seul, fournies par plusieurs unités de santé publique locales de l'Ontario. Par ailleurs, au Nouveau-Brunswick, dans le nord-ouest de l'Ontario et à certains endroits du Manitoba, ont été mis à contribution les deux types de collecte de données : échantillonnage par filet (nécessitant au moins 3 heures-personnes/emplacement) et capture de rongeurs fauniques (moyennant approbation déontologique) (12). Dans toutes les autres études, l'échantillonnage par filet seul a été utilisé.

Les espèces de l'ensemble des tiques relevées dans le cadre des différentes études ont été identifiées par le Laboratoire national de microbiologie de l'Agence de la santé publique du Canada avec des clés d'identification standard. Les emplacements d'échantillonnage ont été cartographiés avec le logiciel ArcGIS Version 10.2 (ESRI). On a calculé la proportion et les intervalles de confiance binomiaux exacts à $95 \%$ des emplacements où $l$. 
scapularis a été observée. Comme ces données n'avaient pas toutes été recueillies de façon simultanée ou par une technique standard quant à la saison et aux efforts d'échantillonnage par filet de traînée, on n'a pas tenté d'effectuer une analyse statistique plus approfondie.

\section{Surveillance passive des tiques}

Ont été géocodés et cartographiés au moyen de ArcGIS version 10.2 (ESRI) les emplacements où est survenue la fixation de tiques soumises par le grand public ou par différents partenaires provinciaux de santé publique rattachés aux cliniques médicales et vétérinaires participantes entre 2004 et 2012. L'analyse n'a porté que sur la soumission de tiques qui s'étaient fixées sur des personnes ou des animaux domestiques ne possédant pas d'antécédents de voyage récent.

\section{Résultats}

\section{Aires endémiques de la maladie de Lyme}

On dénombre présentement 18 aires endémiques de la maladie confirmées et 4 présumées attestant l'établissement de I. scapularis; ces aires se trouvent dans le sud du Manitoba, le sud et l'est de l'Ontario, le sud du Québec, le sud du Nouveau-Brunswick et dans certaines localités de la Nouvelle-Écosse (Figure 2). En outre, certaines de ces aires (le sud du Manitoba, le sud et l'est de l'Ontario, le sud du Québec) comprennent diverses zones particulières (15).

\section{Figure 2: Répartition des aires endémiques de la maladie de Lyme connues (triangles rouges) et} présumées (cercles bleus) au Canada

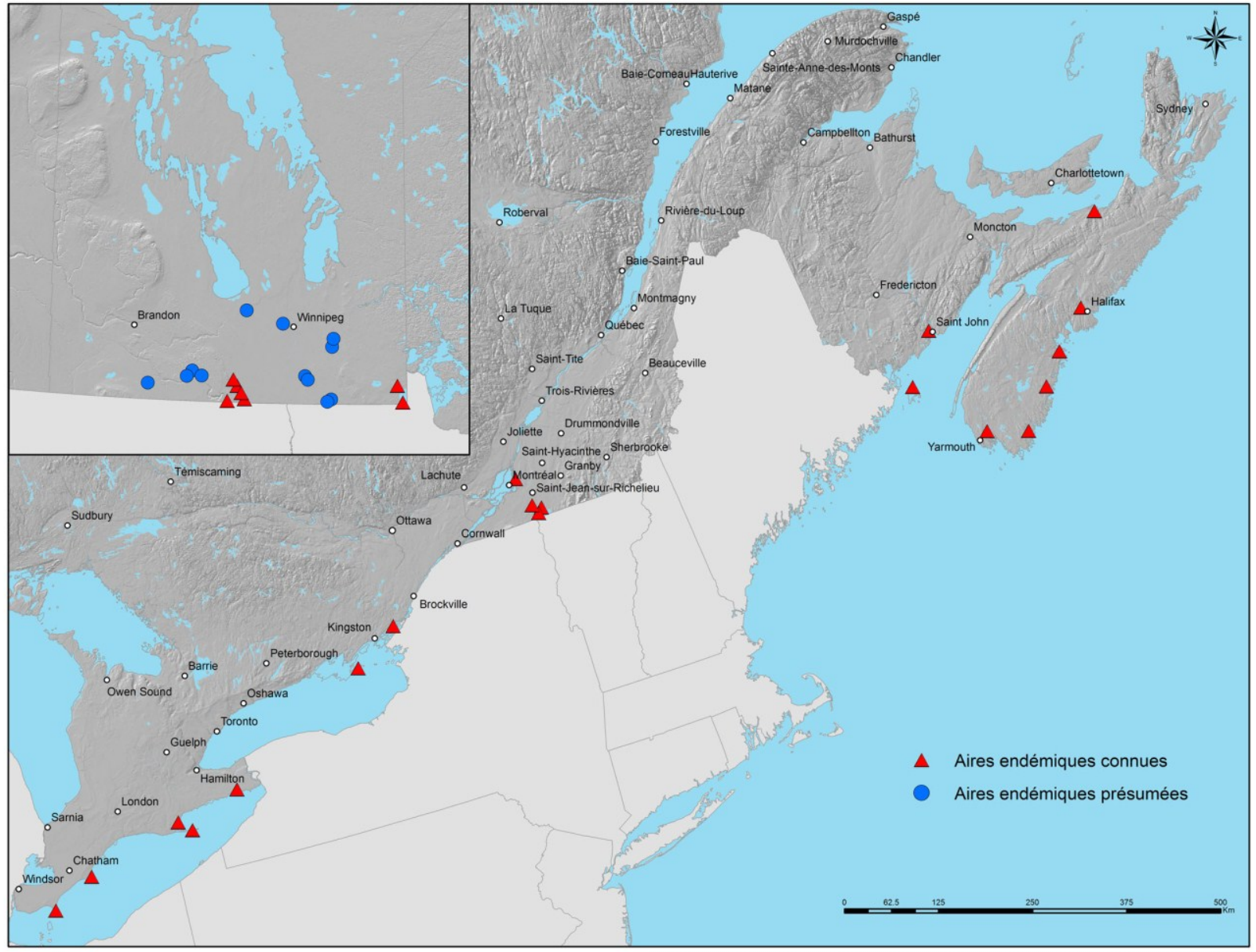




\section{Surveillance sur le terrain}

On a recueilli des données de surveillance sur le terrain à 296 emplacements (70 au Manitoba, 87 en Ontario, 73 au Québec, 16 au Nouveau-Brunswick et 50 en Nouvelle-Écosse) en vue de repérer des populations de $I$. scapularis potentiellement émergentes et un risque éventuel pour la maladie de Lyme (Figure 3).

\section{Figure 3: Résultats des activités de surveillance sur le terrain des tiques à pattes noires au Canada, de 2008 à 2012*}

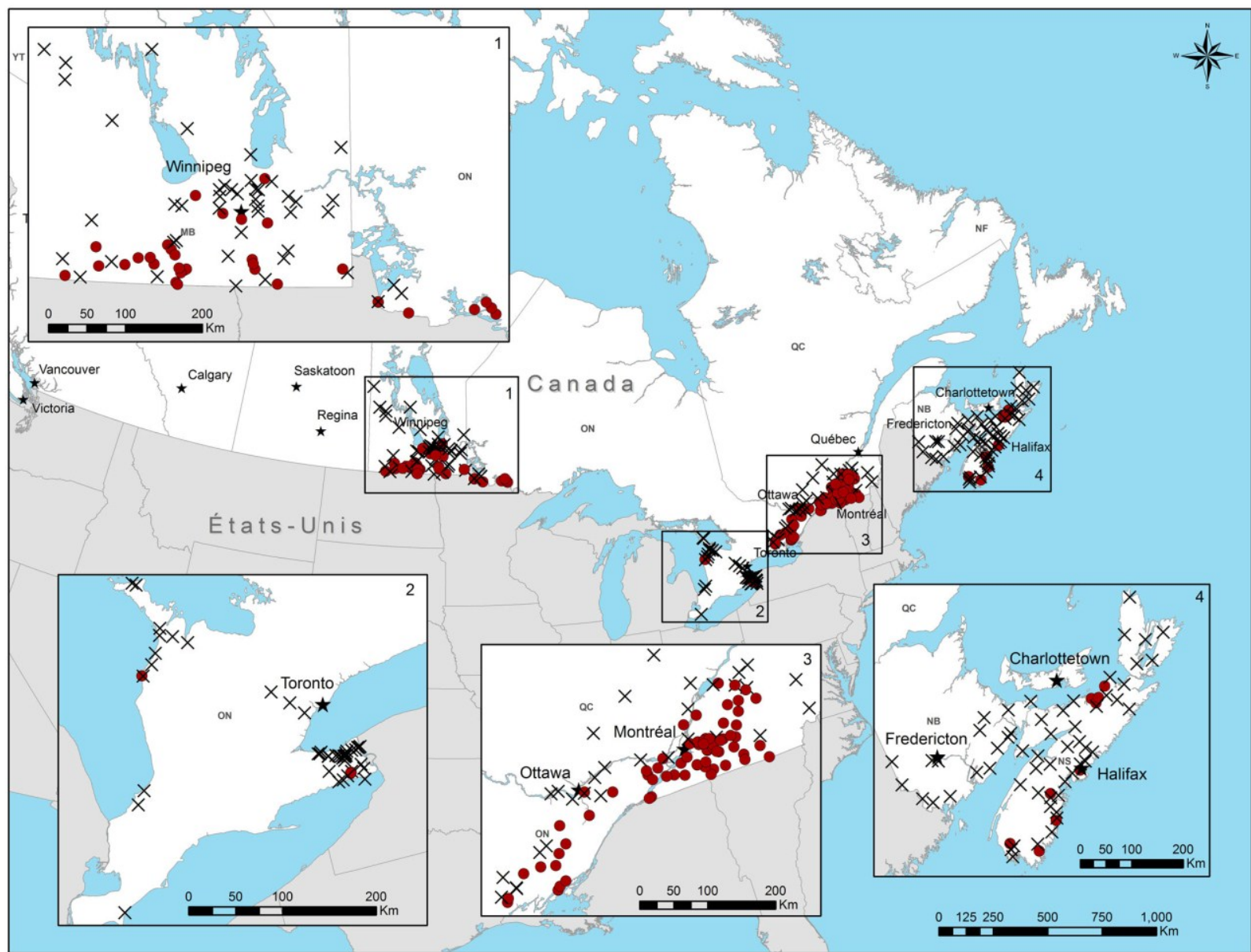

*Les emplacements attestant la présence d'au moins une tique $I$. scapularis sont marqués d'un cercle plein, alors que les emplacements n'attestant pas une telle présence sont marqués d'une croix.

L'étendue des endroits où $I$. scapularis a été repérée varie d'une province à l'autre (Figure 3). On a en effet remarqué cette espèce à bon nombre d'emplacements boisés dans tout le sud du Québec et de l'est de l'Ontario (comprenant effectivement des zones contiguës d'élargissement de l'aire de répartition de I. scapularis), ainsi que dans une région couvrant la partie du Manitoba située au sud de Winnipeg vers le nord-ouest de l'Ontario. Par ailleurs, l'Alberta et la Saskatchewan, à ce que l'on sache, ne sont pas présentement touchées par I. scapularis. 
Nous avons évalué la proportion des emplacements dans une région qui atteste la présence de I. scapularis. La proportion des emplacements positifs pour I. scapularis était sensiblement plus élevée dans le sud du Québec et l'est de l'Ontario que celles des emplacements dans la partie du Manitoba au nord de Winnipeg; dans les régions du Golden Horseshoe et de la péninsule Bruce en Ontario, ainsi qu'au Nouveau-Brunswick et en NouvelleÉcosse (Figure 4).

\section{Figure 4 : Proportion des emplacements visités dans le cadre de la surveillance sur le terrain où la tique $I$. scapularis a été repérée, au Canada*}

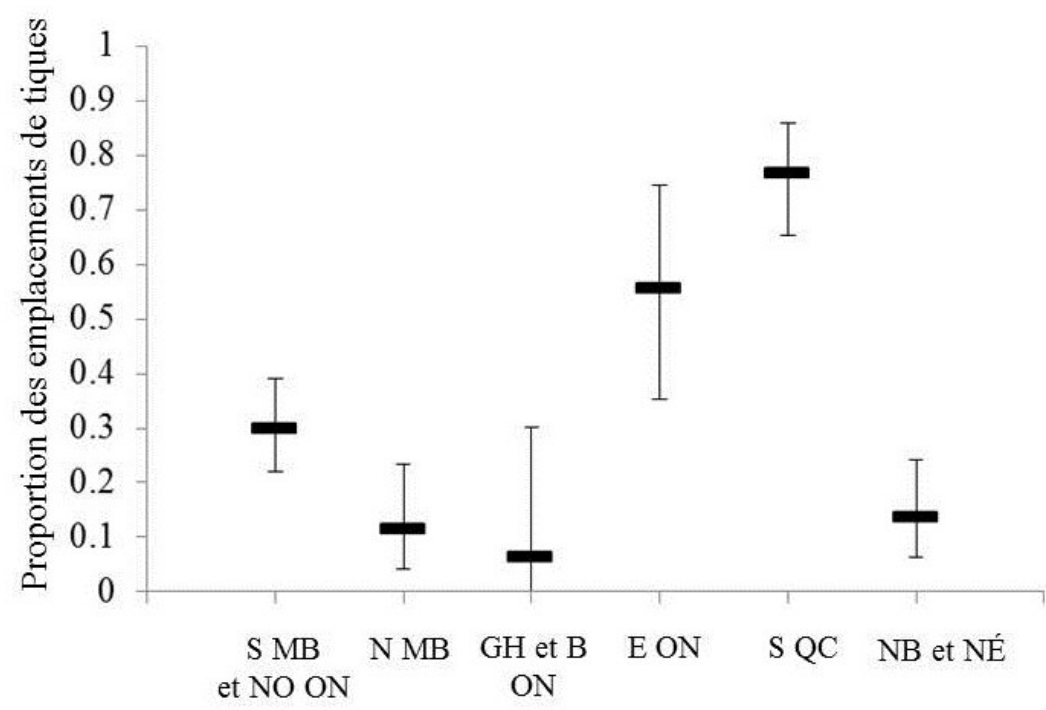

*L'éventail des régions touchées se déploie comme suit : partie du Manitoba au sud de Winnipeg et nord-ouest de I'Ontario (S MB et NO ON); partie du Manitoba au nord de Winnipeg (N MB); régions du Golden Horseshoe et de la péninsule Bruce, en Ontario (GH et B ON), est de l'Ontario (E ON); sud du Québec (S QC); Nouveau-Brunswick et Nouvelle-Écosse (NB et NÉ). À des fins de proportion, les barres d'erreurs indiquent des intervalles de confiance binomiaux exacts à $95 \%$.

\section{Surveillance passive des tiques}

De 2004 à 2012, ont été enregistrées 25738 soumissions, comprenant 28388 tiques particulières, par des cliniques médicales et vétérinaires de 9 provinces (221 soumissions en Alberta, 10 en Saskatchewan, 1063 au Manitoba, 9905 en Ontario, 9371 au Québec, 1631 au Nouveau-Brunswick, 829 à l'île-du-Prince-Édouard, 2553 en Nouvelle-Écosse et 155 à Terre-Neuve). Le nombre de soumissions a ainsi plus que décuplé comparativement aux 2059 soumissions de I. scapularis entre 1990 et 2003 (10). 
Des soumissions survenues entre 2004 et 2012, 569 ne comprenaient que des nymphes; 14, que des larves; 24 925, que des adultes, alors que 230 soumissions comprenaient de nombreuses tiques à plus d'un stade de développement. II n'est pas possible d'effectuer des comparaisons interprovinciales du nombre de tiques soumises en raison d'efforts plus ou moins intenses déployés d'une province à l'autre et au sein d'une même province, ainsi que d'une année à l'autre. La Figure 5 indique les emplacements probables où les tiques ont été attirées par les animaux domestiques ou les humains, puis recueillies.

Figure 5 : Endroits probables où des tiques I. scapularis ont été soumises entre 2004 et 2012 dans le cadre de la surveillance passive (acquises par l'entremise d'animaux domestiques et d'humains grâce à la participation de cliniques médicales et vétérinaires)

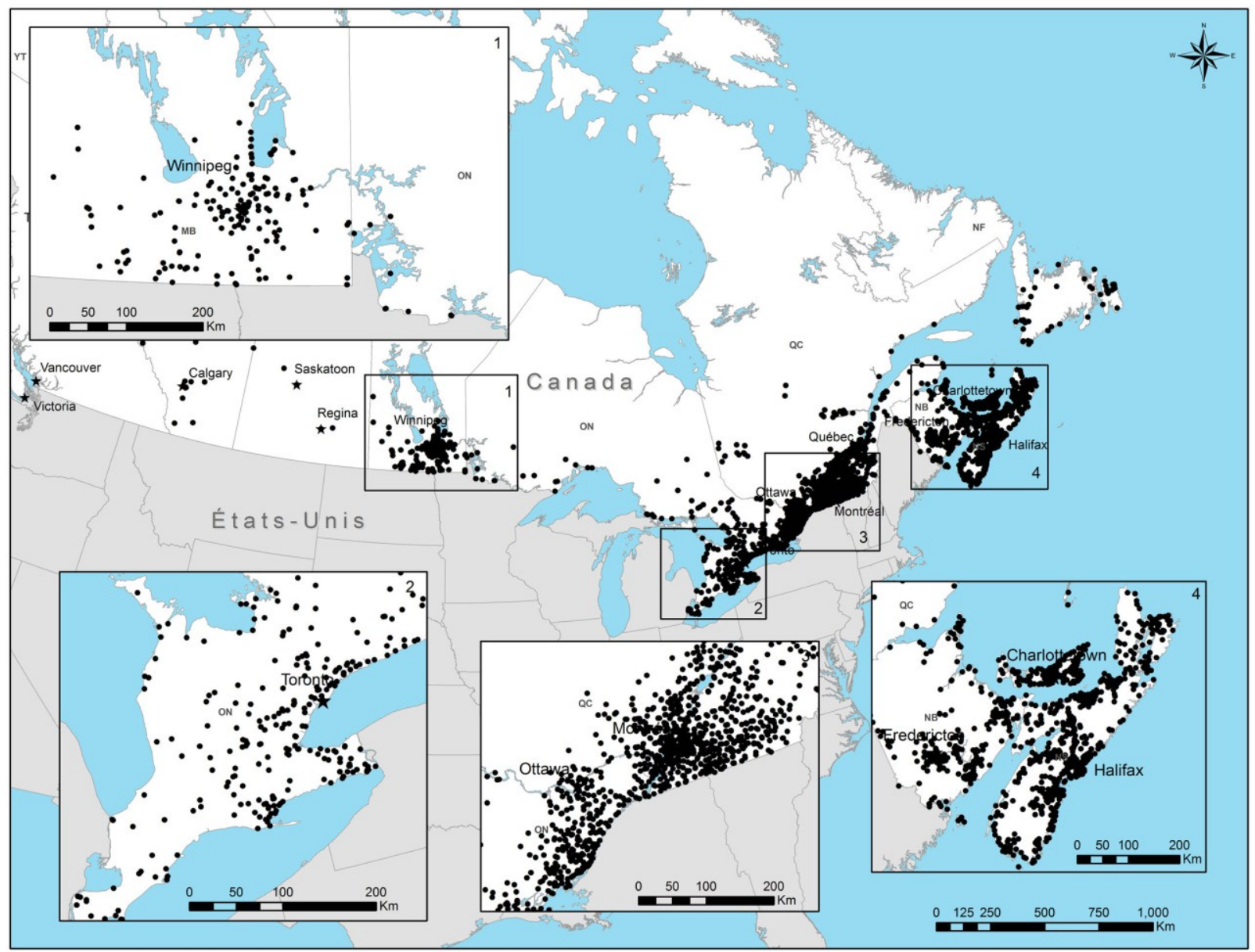

\section{Analyse}

Dans la présente étude, nous avons voulu présenter des données de surveillance sur la tique vectrice $I$. scapularis et sur les aires endémiques de la maladie de Lyme attestant une présence confirmée de $B$. burgdorferi. De tels matériaux indiquent que cette espèce continue à se répandre dans les parties sud de l'est et du centre du Canada.

Les aires endémiques de la maladie de Lyme émergent dans l'est et le centre du pays. Alors qu'en 2009, seulement 10 aires y avaient été confirmées (voir les flèches de la Figure 2) (13), à l'heure actuelle on recense 22 aires endémiques connues ou présumées dans les régions sud de l'est et du centre du Canada. 
Selon les données de surveillance sur le terrain recueillies à partir d'un certain nombre de sources, l'étendue géographique de l'invasion de $I$. scapularis est beaucoup plus vaste que les aires endémiques de la maladie de Lyme comme telles. Or les tendances de l'invasion semblent fluctuer d'un endroit à l'autre. Comme on le soupçonnait, cette invasion survient le plus probablement dans les régions sud du pays avoisinant la frontière américaine, comme l'atteste la surveillance au Manitoba. De vastes zones touchées par l'invasion de I. scapularis surgissent dans les régions contiguës du sud du Manitoba et du nord-ouest de l'Ontario, ainsi que dans les régions contiguës de l'est de l'Ontario et du sud du Québec. Pour ce qui est du sud de l'Ontario à l'ouest de Toronto, du sud du Nouveau-Brunswick et en la Nouvelle-Écosse, exception faite des aires endémiques déjà connues, il y a peu de données démontrant un risque pour la maladie de Lyme aux emplacements visités.

Les divergences géographiques qu'affiche $I$. scapularis sont sans doute attribuables aux variations de la cadence selon laquelle les tiques sont transportées à partir de populations originelles, ainsi qu'aux facteurs facilitant plus ou moins l'établissement de populations de tiques dans une aire donnée (comme le climat, l'habitat et l'abondance d'hôtes fauniques appropriés) $(14,15)$. II reste qu'en général, la surveillance sur le terrain conforte l'exactitude des cartes de risque qui ont été dressées compte tenu des invasions présentes et futures (selon le changement climatique) de I. scapularis (14).

En ce qui concerne les limitations, la présente étude de surveillance en manifeste quelques-unes. En premier lieu, on ne dispose pas actuellement de données pour l'Ouest canadien. Nous savons que la ColombieBritannique compte des aires endémiques de la maladie de Lyme, mais le risque pour cette maladie pourrait être relativement faible du fait que $I$. pacificus est une vectrice moins efficace que $I$. scapularis (13). Ensuite, des limitations surviennent dans les données de surveillance sur le terrain. En effet, il est possible que de faibles densités de tiques se trouvent à certains emplacements qui n'ont pas attesté la présence de tels insectes. Les résultats auraient également pu fluctuer selon le degré d'expérience des agents de terrain, les efforts déployés aux différents emplacements, ainsi que l'année et le mois de surveillance. Enfin, certaines de ces données remontent à 2008 et pourraient donc ne plus correspondre à la situation actuelle.

La répartition des tiques soumises par la surveillance passive s'apparente à celle des populations recensées par la surveillance sur le terrain. Toutefois, on peut également en déduire que le niveau de risque d'exposition à la maladie de Lyme est faible pour les Canadiens habitant dans les régions plus nordiques du Manitoba, de I'Ontario et du Québec, ainsi qu'à Terre-Neuve et à l'île-du-Prince-Édouard. Cet état de choses s'explique probablement par le fait que les tiques sont disséminées à partir d'aires endémiques établies au Canada et aux États-Unis par les oiseaux migrateurs et autres hôtes $(10,16)$. L'évolution du paysage du risque pour la maladie de Lyme au Canada revêt donc diverses formes : nombre grandissant des aires endémiques de la maladie, répartition beaucoup plus vaste de populations de tiques dépistées par la surveillance sur le terrain et décuplement du nombre de $I$. scapularis soumises par surveillance passive. Ces réalités concordent avec la hausse des cas signalés de la maladie de Lyme chez les humains.

Pour ce qui est des recherches à venir, il y aurait lieu de cibler la surveillance intégrée pour les cas humains et les tiques I. scapularis - une telle stratégie permettrait de délimiter les aires à risque pour la maladie de Lyme et de mieux orienter les interventions de santé publique. On procède présentement à la quantification des rapports entre le risque environnemental ainsi que la fréquence et l'incidence des cas humains dans le cadre du programme national de surveillance de la maladie de Lyme. Toutefois, une surveillance élargie du risque environnemental se révélerait une bonne pratique de santé publique, en ce qu'elle permettrait de dépister un risque émergent pour la maladie (et un risque pour d'autres maladies transmises par $I$. scapularis) et d'amorcer des mesures de prévention - et ce, pour prévenir un nombre élevé de cas humains. 


\section{Références}

(1) Kurtenbach K, Hanincova K, Tsao J, Margos G, Fish D et Ogden NH. Key processes in the evolutionary ecology of Lyme borreliosis. Nat Rev Microbiol. 2006;4:660-69.

(2) Bacon RM, Kugeler KJ et Mead PS, Centers for Disease Control and Prevention (CDC). Surveillance for Lyme disease--United States, 1992-2006. MMWR Surveill Summ. 2008;57(SS10):1-9

(3) Thompson C, Spielman A et Krause PJ. Coinfecting deer-associated zoonoses: Lyme disease, babesiosis, and ehrlichiosis. Clin Infect Dis. 2001 33:676-85.

(4) Pritt BS, Sloan LM, Johnson DK, Munderloh UG, Paskewitz SM, McElroy KM et al. Emergence of a new pathogenic Ehrlichia species, Wisconsin and Minnesota, 2009. N Engl J Med. 2011;365:422-9.

(5) Krause PJ, Narasimhan S, Wormser GP, Rollend L, Fikrig E, Lepore T et al. Human Borrelia miyamotoi infection in the United States. N Engl J Med. 2013;368:291-3.

(6) Leighton P, Koffi J, Pelcat Y, Lindsay LR et Ogden NH. Predicting the speed of tick invasion: an empirical model of range expansion for the Lyme disease vector Ixodes scapularis in Canada. J Appl Ecol. 2012;49:45764.

(7) Diuk-Wasser MA, Hoen AG, Cislo P, Brinkerhoff R, Hamer SA, Rowland M et al. Human risk of infection with Borrelia burgdorferi, the Lyme disease agent, in eastern United States. Am J Trop Med Hyg. 2012;86:320-7.

(8) Naleway AL, Belongia EA, Kazmierczak JJ, Greenlee RT et Davis JP. Lyme disease incidence in Wisconsin: a comparison of state-reported rates and rates from a population-based cohort. Am J Epidemiol. 2002;155:1120-7.

(9) Ogden NH, Koffi, JK et Lindsay LR. Évaluation d'un test de dépistage devant servir à déterminer le risque de la maladie de Lyme. RMTC 2014, vol. 40-5.

(10) Ogden NH, Trudel L, Artsob H, Barker IK, Beauchamp G, Charron D et al. Ixodes scapularis ticks collected by passive surveillance in Canada: analysis of geographic distribution and infection with the Lyme borreliosis agent Borrelia burgdorferi. J Med Entomol. 2006;43:600-9.

(11) Santé Canada. Consensus conference on Lyme disease. CMAJ. 1991;144:1627-32.

(12) Bouchard C, Beauchamp G, Nguon S, Trudel L, Milord F, Lindsay LR et al. Associations between Ixodes scapularis ticks and small mammal hosts in a newly endemic zone in southeastern Canada: implications for Borrelia burgdorferi transmission. Ticks Tick Borne Dis. 2011;2:183-90.

(13) Ogden NH, Lindsay LR, Morshed M, Sockett PN et Artsob H. The emergence of Lyme disease in Canada. CMAJ. 2009;180:1221-4

(14) Ogden NH, St-Onge L, Barker IK, Brazeau S, Bigras-Poulin M, Charron DF et al. Risk maps for range expansion of the Lyme disease vector, Ixodes scapularis, in Canada now and with climate change. Int J Health Geogr. 2008;7:24.

(15) Ogden NH, Mechai S et Margos G. Changing geographic ranges of ticks and tick-borne pathogens: drivers, mechanisms and consequences for pathogen diversity. Front Cell Infect Microbiol. 2013;3:46.

(16) Ogden NH, Bouchard C, Kurtenbach K, Margos G, Lindsay LR, Trudel L et al. Active and passive surveillance and phylogenetic analysis of Borrelia burgdorferi elucidate the process of Lyme disease risk emergence in Canada. Environ Health Perspect. 2010;118:909-14. 


\section{Remerciements}

Nous tenons à remercier nos collègues des organismes provinciaux suivants pour leur participation au réseau de surveillance passive et sur le terrain de tiques : Santé Alberta; Santé Saskatchewan; Santé Manitoba; l'Agence ontarienne de protection et de promotion de la santé; l'Institut national de santé publique du Québec; le Laboratoire de santé publique du Québec; I'Université de Montréal; Agriculture, Aquaculture et Pêches NouveauBrunswick ainsi que Santé Nouveau-Brunswick; le ministère de la Santé et du Bien-être, ainsi que le ministère des Ressources naturelles de la Nouvelle-Écosse; enfin le ministère des Ressources naturelles de Terre-Neuveet-Labrador). Nous remercions également les comtés de Hastings et de Prince Edward, de Chatham-Kent, les régions de Peel et de Niagara, le comté de Lambton, ainsi que les unités sanitaires Grey Bruce et Northwestern, en Ontario, qui ont fourni des données de surveillance sur le terrain. Nous reconnaissons également la contribution des vétérinaires ainsi que nombre de personnes issues du milieu universitaire et du grand public au Canada qui ont soumis des tiques.

\section{Déclaration de conflit d'intérêts}

II n'y a aucun conflit d'intérêts à déclarer.

\section{Financement}

Ce travail a été appuyé par l'Agence de la santé publique du Canada. 